Article

\title{
A Haar Wavelet Decision Feedback Channel Estimation Method in OFDM Systems
}

\author{
Ruiguang Tang ${ }^{(D)}$, Xiao Zhou * (iD) and Chengyou Wang \\ School of Mechanical, Electrical and Information Engineering, Shandong University, Weihai 264209, China; \\ trg@mail.sdu.edu.cn (R.T.); wangchengyou@sdu.edu.cn (C.W.) \\ * Correspondence: zhouxiao@sdu.edu.cn; Tel.: +86-631-568-8338
}

Received: 16 April 2018; Accepted: 22 May 2018; Published: 26 May 2018

check for updates

\begin{abstract}
Channel estimation is a key technology in improving the performance of the orthogonal frequency division multiplexing (OFDM) system. The pilot-based channel estimation method decreases the spectral efficiency and data transmission rate. Some conventional channel estimation methods cannot suppress the noise effectively, which affects the quality of the final received signals. To solve these two problems, a Haar wavelet decision feedback (DF) channel estimation method, also named the Haar wavelet method, is proposed in this paper. The proposed Haar wavelet method can suppress the noise existing at the estimated channel impulse response (CIR) effectively, based on a time-domain threshold which is a standard deviation of noise obtained by wavelet decomposition. At the same time, the proposed Haar wavelet method just requires inserting one block pilot symbol in front of the first OFDM symbol, which therefore improves the data transmission rate and spectrum efficiency greatly. Simulation results are shown to verify the effectiveness of the proposed Haar wavelet method in multipath channel propagation conditions.
\end{abstract}

Keywords: channel estimation; orthogonal frequency division multiplexing (OFDM); decision feedback (DF); wavelet decomposition

\section{Introduction}

Orthogonal frequency division multiplexing (OFDM) system [1-5] has been widely used in modern wireless communication systems due to its high spectrum efficiency and strong multipath fading resistance ability. The cyclic prefix (CP) is used as the guard interval in each OFDM symbol to reduce the inter-symbol interference (ISI) and inter-carrier interference (ICI) greatly. At the same time, the OFDM system utilizes the time delay characteristics of multipath fading channel which greatly simplifies the equalizer design. The OFDM technology can also combine with the multiple-input multiple-output (MIMO) technology to enhance the channel capacity [6]. The wireless channel is an important part in OFDM systems. But when the signal passes through the wireless fading channel, it will be disturbed by the additive white Gaussian noise (AWGN) which affects the quality of signal transmission and the performance of the system. Some papers $[7,8]$ use Turbo coding with code rate of $1 / 3$ and $1 / 2$ respectively at the transmitter in OFDM system to suppress the effect of noise in wireless channel. It can obtain good performance in bit error rate (BER). To further improve the OFDM system performance, channel estimation is a key technology, and an efficient channel estimation method can greatly improve the system performance.

To estimate the characteristics of the channel, some conventional channel estimation methods are often performed in frequency-domain using pilots [9], such as least square (LS) method [10-12], minimum mean square error (MMSE) method [13], linear MMSE (LMMSE) method [14], singular value decomposition (SVD) method [15], noise suppression threshold method [16,17], and so on. The LS channel estimation method has the advantage of low complexity and easy implementation. But this 
method does not consider any noise effect in obtaining its solutions and has poor performance [10,11]. In [12], LS channel estimation method is applied on the space-time block-code based MIMO-OFDM system. It also designs a new pilot pattern to further improve the accuracy of the channel estimation. This method has low complexity, but it may has poor performance in dynamic channel which restrains the application. The MMSE channel estimation method has good performance because of utilizing the channel statistics and the signal-to-noise ratio (SNR) to suppress the effect of noise. However this method requires a large amount of calculations concerning the inverse operation of the matrix, and the hardware implementation is relatively hard [13]. To further reduce the calculation complexity, the LMMSE channel estimation method is proposed in [14]. But the disadvantage of this method is that much priori information is required to calculate the autocorrelation matrix about the channel. Hence, the application of this method will be restrained in complicated fading channel conditions. In [15], the discrete cosine transform-SVD (DCT-SVD) based channel estimation technique was presented in IEEE 802.16e downlink partially used sub channelization system. This channel estimation method has a low calculation complexity. However, it just utilizes the outer coding technology in downlink partially used sub channelization system, and the channel estimation accuracy is decreased in fast fading environment. A time-domain threshold channel estimation method for OFDM systems was proposed in [16]. This method is based on a time-domain threshold which is a standard deviation of noise obtained by wavelet decomposition. To obtain a good performance, the time-domain threshold decreases with the increase of SNR. But the disadvantage of this method is that it uses comb pilots to estimate the channel state information which greatly reduces the spectrum efficiency. In [17], the linear filter least square channel estimation method was proposed in OFDM systems. This method can effectively suppress the AWGN by the linear filter in time-domain. But the suppression factor of the linear filter in this paper is usually utilized in time-domain synchronous-OFDM (TDS-OFDM) systems. Hence, other communication systems, such as underwater acoustic communication and MIMO-OFDM systems, may require choosing a new suppression factor to effectively suppress the AWGN.

The pilot-based channel estimation method is relied on the pilot symbols to estimate the channel state information which reduces the data transmission rate and spectrum efficiency. Another channel estimation method which has been extensively studied is called blind channel estimation method [18-20]. The blind channel estimation method presented in [21,22] requires no pilots to estimate the channel state information. Hence, the blind channel estimation method has higher spectrum and data transmission efficiency than the pilot-based channel estimation methods. But at the same time, the blind channel estimation method requires calculating the statistical properties between the transmitted symbols and received symbols to estimate the channel impulse response (CIR). Hence, this method has high computational complexity which restrains the application in the OFDM systems. To further reduce the computation complexity of blind channel estimation method, a precoding-assisted blind channel estimation algorithm was proposed in [23]. This algorithm designs a non-redundant linear pre-coder based on auto-correlation matrix at the transmitter. This method has low complexity than conventional subspace-based blind channel estimation method. But this algorithm cannot be applied in MIMO system which is widely used today.

The semi-blind channel estimation method is a tradeoff between computational complexity and spectrum efficiency [24-26]. Compared with the pilot-based channel estimation method, the semi-blind method uses less pilots to estimate the channel state information which improves the data transmission rate and spectrum efficiency. At the same time, the computational complexity is further reduced compared with the blind channel estimation method. In [27], a quasi-Newton method for semi-blind estimation is derived for channel estimation in uplink cloud radio access networks. Firstly, the initial channel state information is provided by LS channel estimation. Then, the maximum-likelihood principle is used to improve the estimation performance in the semi-blind channel estimation. Simulation results show that semi-blind channel estimation decreases the overhead of bandwidth without sacrificing the mean square error (MSE). In [28], an iterative space-alternating generalized expectation maximization (SAGE) based semi-blind channel estimation method was presented in 
massive MIMO systems. This method updates the pilot-based channel estimate errors with the help of SAGE algorithm. It first uses a few pilots to obtain the initial channel parameters. Then, the iteratively SAGE algorithm is used to improve the accuracy of the initial channel parameters. The advantage of this method is that it saves the additional pilot overhead and achieves convergence in almost one iteration. But the disadvantage of this method is that the SAGE algorithm increases the system complexity. An optimized semi-blind channel estimation method in OFDM systems was presented in [29]. This method uses the scaled LS technique to estimate channel state information and further eliminate the subspace decomposition estimation error which is caused by the noise in the channel. Due to using a few pilots, this method improves the spectrum efficiency. But the disadvantage of this method is that it cannot suppress the ICI and ISI effectively due to using the zero prefix (ZP) as the guard interval. In paper [30], a semi-blind algorithm that uses the linear prediction as the blind channel estimation, in combination with some training data to further improve the channel estimation accuracy, yielding a better estimation performance. However, this semi-blind estimation algorithm is not applicable in MIMO-OFDM systems, since the signal model in MIMO-OFDM systems is quite different from that in MIMO systems.

This paper proposes a Haar wavelet decision feedback (DF) channel estimation method applied in wavelet-OFDM system. It has high spectrum and data transmission efficiency while suppressing the AWGN existing at the estimated channel coefficients in time-domain effectively. It can improve the system BER and normalized MSE (NMSE) performance and is easy to implement in the system hardware. Simulation results verify that the proposed Haar wavelet method is superior to the conventional LS, discrete Fourier transform (DFT), and DF channel estimation methods in terms of BER and NMSE performance under multipath fading channel conditions.

The remainder of this paper is organized as follows. In Section 2, the system model is presented. Section 3 introduces three conventional channel estimation methods. Section 4 illustrates the proposed Haar wavelet decision feedback channel estimation method. The experimental results and comparison analysis are presented in Section 5. Conclusions and future work are presented in Section 6.

\section{System Model}

The system model of the wavelet-OFDM system is presented in Figure 1 [17].

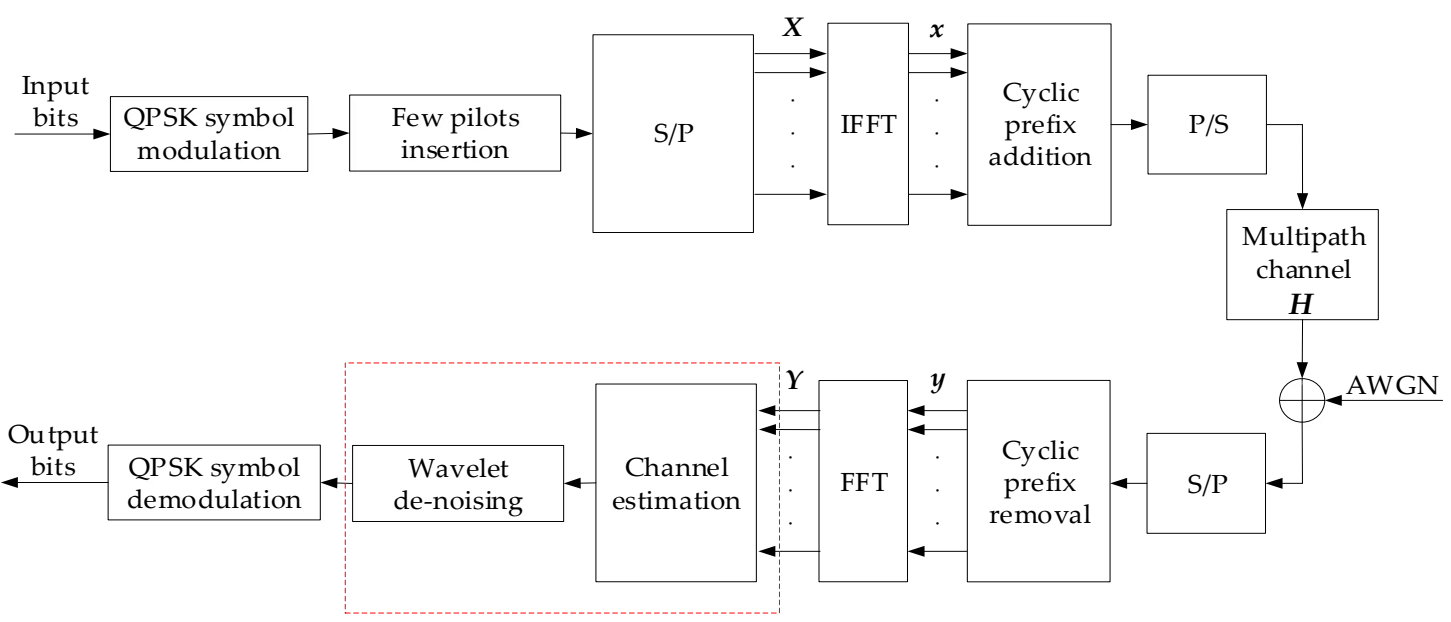

Figure 1. System model of the wavelet-orthogonal frequency division multiplexing (OFDM) system.

At the transmitter, the incoming binary information is first grouped and mapped according to a pre-specified modulation scheme-quadrature phase shift keying (QPSK). After inserting few pilots, the QPSK symbols are converted into parallel streams by serial-to-parallel block. Inverse fast Fourier transform (IFFT) block is used to transform the data sequence $X$ with $N$ rows into time-domain signal, where $N$ is the number of subcarriers in frequency-domain. Then, the $\mathrm{CP}$ is inserted into the 
time-domain OFDM symbols $x$ in order to reduce the ICI and ISI. The transmitted signal will pass through the multipath fading channel with AWGN after parallel to serial block.

At the receiver, the $\mathrm{CP}$ is removed after the serial to parallel conversion. Following the fast Fourier transform (FFT), the estimated channel state information is obtained in the channel estimation block. The AWGN existing at the estimated channel coefficients is further reduced in the wavelet de-noising block. The detail process of dotted line block in Figure 1 is described in Section 4. At last, the binary information data is obtained after the demodulation with QPSK demodulation scheme.

\section{Conventional Channel Estimation Methods}

\subsection{LS Channel Estimation}

The LS channel estimation method can be easy to implement in OFDM systems [11]. In LS channel estimation method, block type pilots are used to estimate the channel state information. It can be shown that the estimated channel frequency response (CFR) at pilots by the LS method is represented as

$$
\hat{\boldsymbol{H}}_{\mathrm{LS}}\left(k_{\mathrm{P}}\right)=\frac{\boldsymbol{Y}\left(k_{\mathrm{P}}\right)}{\boldsymbol{X}\left(k_{\mathrm{P}}\right)}
$$

where $\boldsymbol{X}\left(k_{\mathrm{P}}\right)$ and $\boldsymbol{Y}\left(k_{\mathrm{P}}\right)$ are the transmitted and received pilot signal on the $k_{\mathrm{P}}$-th pilot symbol, respectively. The channel coefficients of all data symbols in frequency-domain $\hat{\boldsymbol{H}}_{\mathrm{LS}}$ can be obtained by one-dimensional linear interpolation.

\subsection{DFT Channel Estimation}

Although LS channel estimation method is relatively simple and easy to implement, the significant disadvantage of the method is that it is susceptible to the AWGN of the channel. The DFT channel estimation method is carried out in time-domain to suppress the effect of noise. The channel response values beyond the length of guard interval are set to zero to remove the impact of AWGN in this method. The procedure of the DFT channel estimation method is shown in Figure $2[17,31]$.

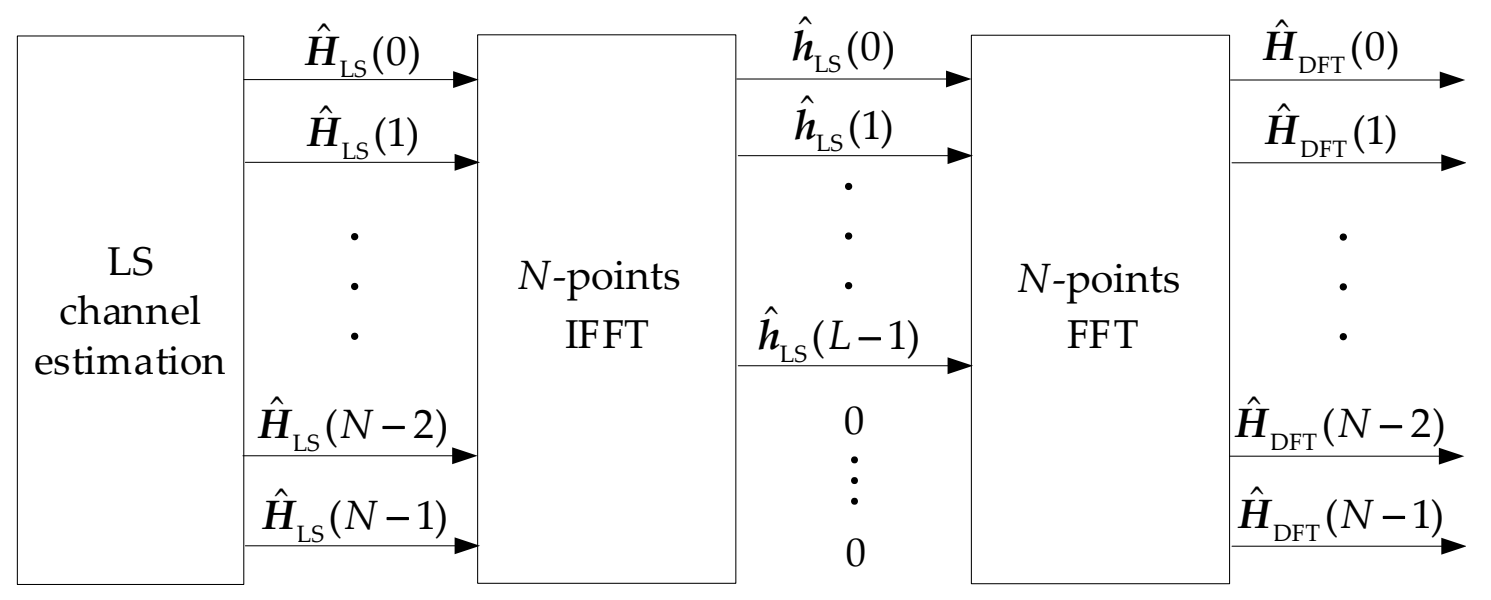

Figure 2. Discrete Fourier transform (DFT) channel estimation method in cyclic prefix (CP)-OFDM system.

As shown in Figure 2, the CFR $\hat{H}_{\text {LS }}$ obtained by the LS channel estimation method is converted into time-domain CIR $\hat{h}_{\mathrm{LS}}(n)$ with $n=0,1,2, \cdots, N-1$ by the IFFT operation. Assuming that $L$ is the number of points of guard interval, the length of CIR should be no longer than that of the guard interval. To suppress the AWGN effectively, $\hat{h}_{\mathrm{LS}}(n)$ should be considered to be AWGN when $n \geq L$. After noise suppression, the estimated CIR $\hat{h}_{\mathrm{DFT}}(n)$ can be expressed as [17] 


$$
\hat{h}_{\mathrm{DFT}}(n)=\left\{\begin{array}{cc}
\hat{h}_{\mathrm{LS}}(n), & 0 \leq n<L, \\
0, & n \geq L .
\end{array}\right.
$$

At last, the new noise removal CFR $\hat{H}_{\mathrm{DFT}}$ can be obtained by the FFT operation of $\hat{\boldsymbol{h}}_{\mathrm{DFT}}(n)$.

\subsection{DF Channel Estimation}

Due to using a large number of pilots in the LS and DFT channel estimation methods, the data transmission rate and spectrum efficiency of the system are decreased in a certain extent. The DF channel estimation method does not require transmitting the block pilots regularly within a certain time interval. It just requires inserting one block pilot symbol in front of the first OFDM symbol to estimate the initial CFR. The block type pilot is distributed on each subcarrier uniformly. channel state information can be obtained through the process of iterative channel estimation. For high speed data communication system, this method improves the communication efficiency. The procedure of the DF channel estimation method is shown in Figure 3 [32].

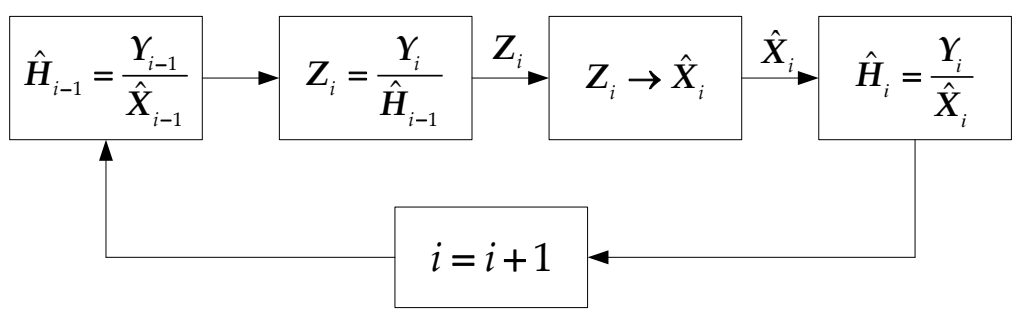

Figure 3. Decision feedback (DF) channel estimation method in CP-OFDM system.

To reduce the use of pilots, the DF channel estimation method uses the channel state information of current OFDM symbol as the next OFDM symbol's channel coefficients. In the process of iterative channel estimation, this method requires inserting one block pilot symbol to obtain the initial channel state information by LS method which is presented as

$$
\hat{H}_{\mathrm{P}}=\frac{Y_{\mathrm{P}}}{X_{\mathrm{P}}}
$$

where $Y_{P}$ and $X_{P}$ are the received and transmitted block type pilot symbols, respectively. The equilibrium value of the first OFDM symbol $Z_{1}$ can be obtained by the CFR $\hat{\boldsymbol{H}}_{\mathrm{P}}$ which is presented as

$$
Z_{1}=\frac{Y_{1}}{\hat{H}_{\mathrm{P}}}
$$

where $\boldsymbol{Y}_{1}$ is the first received OFDM symbol. As shown in Figure 3 , the previously estimated CFR $\hat{\boldsymbol{H}}_{i-1}$ is taken as a prediction of the current channel coefficients. The equilibrium value of the current OFDM symbol $Z_{i}$ is obtained by Equation (5) which is presented as

$$
\mathbf{Z}_{i}=\frac{Y_{i}}{\hat{\mathbf{H}}_{i-1}},
$$

where $i$ is the index of OFDM symbols. Then, the estimated current OFDM symbol's transmission value $\hat{X}_{i}$ can be obtained by hard decision. The detected symbol $\hat{X}_{i}$ will be further divided by the received symbol $Y_{i}$ in order to calculate the new estimated CFR $\hat{H}_{i}$ which is represented as

$$
\hat{\boldsymbol{H}}_{i}=\frac{Y_{i}}{\hat{\boldsymbol{X}}_{i}} .
$$


From Equations (3) to (6), the division means the corresponding element division between two vectors. The channel coefficients of all data symbols in frequency-domain $\hat{\boldsymbol{H}}_{\mathrm{DF}}$ are obtained through the iterative process above. At the same time, the transmitted symbol $\hat{X}_{i}$ can be obtained during the iterative channel estimation.

\section{Proposed Haar Wavelet Decision Feedback Channel Estimation Method}

To have high spectrum and data transmission efficiency like the DF channel estimation method, at the same time, the AWGN in time-domain can be effectively removed. This paper proposes a Haar wavelet decision feedback channel estimation method. The threshold obtained by the Haar wavelet decomposition can be utilized to suppress the AWGN in time-domain effectively. Compared with the time-domain threshold channel estimation method in [16] which also uses Haar wavelet, this paper uses DF channel estimation method to obtain the initial channel estimation coefficients and improves the spectrum and data transmission rate. Paper [16] uses pilot-based LS method to obtain the initial channel estimation coefficients which decreases the spectrum and data transmission efficiency. In the wavelet-OFDM system proposed in this paper, IFFT and FFT operations are used to combine different subcarriers into a composite time-domain signal. But papers [33-35] use inverse discrete wavelet transform (IDWT) and DWT instead of IFFT and FFT, which aim to suppress the ICI and ISI. Paper [35] also investigated different wavelets to adapt to the specific channel environment. The Haar wavelet is used as subcarriers in [33-35]. In this paper, Haar wavelet decomposition is used for suppressing noise existing at the estimated channel coefficients in time-domain.

In the practical application, the received signals are disturbed by the AWGN. After the wavelet decomposition, signals are divided into the important and unimportant coefficients [16]. The important coefficients contain the useful information about the signal and the AWGN is concentrated on the unimportant coefficients. The important coefficients also called approximation coefficients can be obtained based on low-pass filter. The unimportant coefficients also called detail coefficients can be obtained based on high-pass filter. The Haar wavelet has the advantage of simple and easy implementation. In addition, the channels used in this paper are multipath fading channels which have the characteristic of frequency selectivity. Haar wavelet is more suitable for this channel characteristic [36]. Hence, this paper uses Haar wavelet to decompose the channel response. According to Haar wavelet, the frequency response of low-pass filter $F\left(\mathrm{e}^{\mathrm{j} \omega}\right)$ and high-pass filter $G\left(\mathrm{e}^{\mathrm{j} \omega}\right)$ can be respectively expressed as [16]

$$
\begin{gathered}
F\left(\mathrm{e}^{\mathrm{j} \omega}\right)=\mathrm{e}^{\mathrm{j} \omega / 2} \cos \left(\frac{\omega}{2}\right), \\
G\left(\mathrm{e}^{\mathrm{j} \omega}\right)=-\mathrm{j} \mathrm{e}^{\mathrm{j} \omega / 2} \sin \left(\frac{\omega}{2}\right),
\end{gathered}
$$

where $\omega$ is radian frequency.

The detail coefficients are obtained by high-pass filter and the frequency of noise is relatively higher than that of the signal. As a result, noise existing at high frequency is the dominant components in the detail coefficients. The standard deviation of the detail coefficients corresponding to the noise added to the signal can be obtained by [16]

$$
\hat{\sigma}=\frac{\operatorname{median}\left(\left|\boldsymbol{d}_{i}\right|\right)}{0.3045}
$$

where $\boldsymbol{d}_{i}$ are the detail coefficients obtained by the Haar wavelet decomposition. median $(\cdot)$ is the operation of obtaining the mid-value of a vector. In [16], constant value 0.6745 is used as the denominator in Equation (9). The channel environment used in this paper is different from the paper [16]. Simulation verifies that 0.3045 is an optimal value to obtain good system performance. The procedure of the proposed Haar wavelet method is presented in Figure 4. 


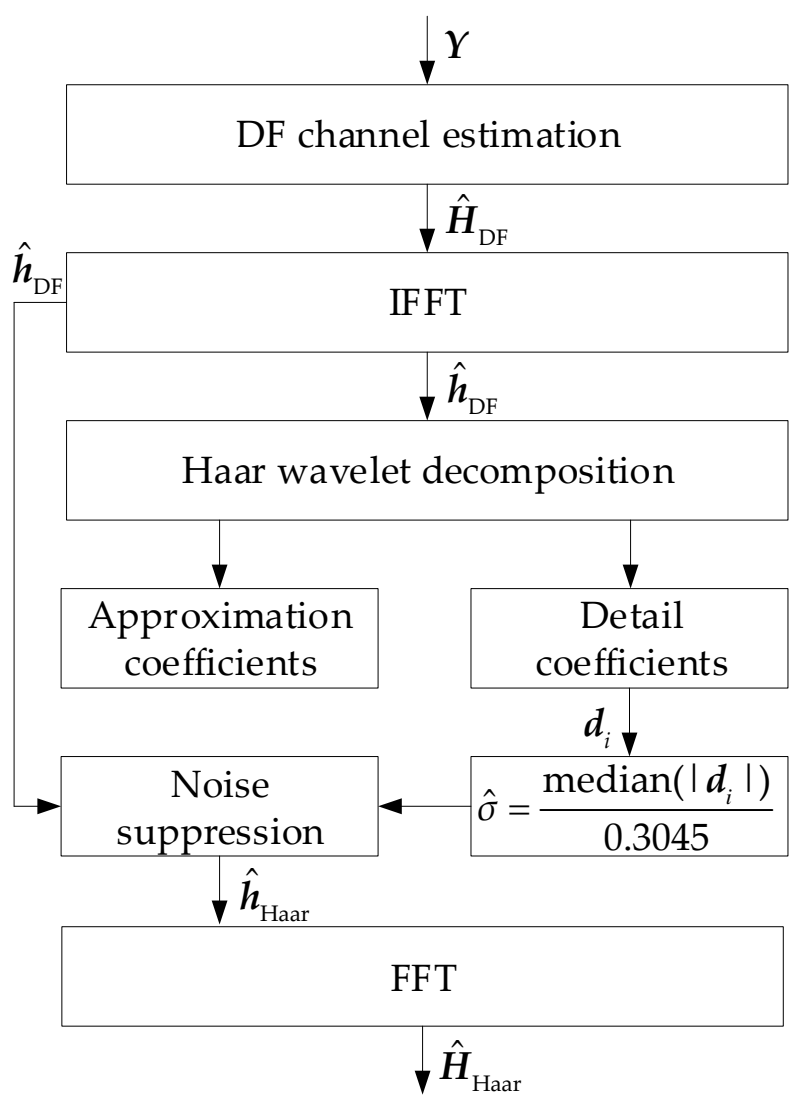

Figure 4. The proposed Haar wavelet channel estimation method in wavelet-OFDM system.

As shown in Figure 4, to obtain high data transmission rate and spectrum efficiency, the initial CFR $\hat{H}_{\mathrm{DF}}$ is obtained by the DF channel estimation method. Then, the time-domain CIR $\hat{h}_{\mathrm{DF}}$ is obtained by the IFFT operation. After the IFFT operation, the Haar wavelet decomposition is performed to obtain the detail coefficients $\boldsymbol{d}_{i}$. Based on the Equation (9), the $\hat{\sigma}$ is obtained as the threshold to reduce the AWGN in time-domain which is presented as

$$
\hat{\boldsymbol{h}}_{\mathrm{Haar}}(n)=\left\{\begin{array}{cl}
\hat{\boldsymbol{h}}_{\mathrm{DF}}(n), & \hat{\boldsymbol{h}}_{\mathrm{DF}}(n) \geq \hat{\sigma}, \\
0, & \hat{\boldsymbol{h}}_{\mathrm{DF}}(n)<\hat{\sigma} .
\end{array}\right.
$$

After setting the insignificant channel coefficients that below the threshold to be zero, the noiseremoved channel coefficients are transformed into frequency-domain coefficients $\hat{\boldsymbol{H}}_{\mathrm{Haar}}$ in the FFT block. Finally, through these procedures, the proposed Haar wavelet method improves the data transmission rate and spectrum efficiency while suppressing the AWGN effectively.

\section{Simulation Results}

This section presents the BER and NMSE performance comparisons between LS, DFT, DF, and the proposed Haar wavelet decision feedback method. The simulation is performed in static and dynamic multipath environments, respectively. The multipath channel models are China DTV Test 1st (CDT1) and China DTV Test 6st (CDT6). CDT1 and CDT6 channels are from the field tests for digital television terrestrial broadcasting standard [37]. Both CDT1 and CDT6 channels are belong to the Rayleigh fading channel. Under Rayleigh fading channel environment, the received signals suffer from the effect of multipath propagation. The received signals come from the different paths and have different delay time before arriving at the receiver. It is assumed that the components of the received waveform obey 
the Gaussian distribution with a mean value of zero and standard deviations $\sigma_{\mathrm{R}}^{2}$, and the probability density function of the Rayleigh fading amplitude is presented as [8]

$$
f(a)=\left\{\begin{array}{cc}
\frac{a}{\sigma_{\mathrm{R}}^{2}} \exp \left(-\frac{a}{2 \sigma_{\mathrm{R}}^{2}}\right), & a \geq 0 \\
0, & a<0
\end{array}\right.
$$

where $a$ is the amplitude of the received signal. CDT1 is a typical multipath broadcasting channel model which has slight frequency-selectivity. CDT6 channel has stronger frequency-selectivity than CDT1 channel. These two channels are used to verify the effectiveness to channel characteristics of the proposed Haar wavelet method.

The profiles for the CDT1 and CDT6 channel models are shown in Table 1. The main simulation parameters based on wavelet-OFDM system and CP-OFDM system are presented in Table 2.

Table 1. Profiles for China DTV Test 1st (CDT1) and CDT6 multipath fading channels [17].

\begin{tabular}{ccccc}
\hline \multirow{2}{*}{ Tap } & \multicolumn{2}{c}{ CDT1 } & \multicolumn{2}{c}{ CDT6 } \\
\cline { 2 - 5 } & Delay $(\boldsymbol{\mu s})$ & Power $(\mathbf{d B})$ & Delay $(\boldsymbol{\mu s})$ & Power $(\mathbf{d B})$ \\
\hline 1 & 0 & 0 & 0 & 0 \\
2 & -1.8 & -20 & -18 & -10 \\
3 & 0.15 & -20 & -1.8 & -20 \\
4 & 1.8 & -10 & 0.15 & -20 \\
5 & 5.7 & -14 & 1.8 & -10 \\
6 & 18 & -18 & 5.7 & -14 \\
\hline
\end{tabular}

Table 2. Parameters for wavelet-OFDM and CP-OFDM systems.

\begin{tabular}{cc}
\hline Parameters & Specifications \\
\hline System model & wavelet-OFDM/CP-OFDM \\
Modulation mode & QPSK \\
CP length (subcarriers) & $128 / 140$ \\
Subcarrier number & 512 \\
OFDM block length & 10 \\
Doppler spread & $20 \mathrm{~Hz}$ \\
\hline
\end{tabular}

The conventional LS, DFT, and DF channel estimation methods are presented in CP-OFDM system. The proposed Haar wavelet method is proposed in wavelet-OFDM system. In the wavelet-OFDM and CP-OFDM systems, the modulation mode is the QPSK scheme. The total number of data subcarriers is 512. The CP occupies 128 subcarriers in the simulation of CDT1 channel. Due to CDT6 channel has stronger frequency-selectivity than CDT1 channel, this paper adds the length of CP under CDT6 channel environment. The $\mathrm{CP}$ occupies 140 subcarriers in the simulation of CDT6 channel. One OFDM block consists of 10 OFDM symbols. In LS and DFT channel estimation methods, 5 block type pilots are inserted into the OFDM symbols uniformly. In DF and the Haar wavelet methods, one block pilot symbol is inserted into the front of the first OFDM symbol. This paper utilizes the Doppler spread equivalent to $20 \mathrm{~Hz}$ in dynamic multipath channel environment. The threshold $\hat{\sigma}$ obtained in the proposed Haar wavelet method under CDT1 and CDT6 channels are presented in Figure 5.

As can be seen from Figure 5, with the increase of SNR, the threshold $\hat{\sigma}$ under both CDT1 and CDT6 channels are decreasing, respectively. Under low SNR conditions, noise has larger energy than the useful signal. Hence, the threshold $\hat{\sigma}$ is set to be large to suppress the noise to the maximum extent. According to Equation (9), when SNR $=10 \mathrm{~dB}$, the thresholds $\hat{\sigma}$ are 0.0268 and 0.0281 in CDT1 and CDT6 channels, respectively. When SNR $=14 \mathrm{~dB}$, the thresholds $\hat{\sigma}$ are 0.0148 and 0.0164 in CDT1 and CDT6 channels, respectively. The changing threshold can filter the noise effectively and improve the accuracy of channel estimation, reducing the system BER at the same time. 


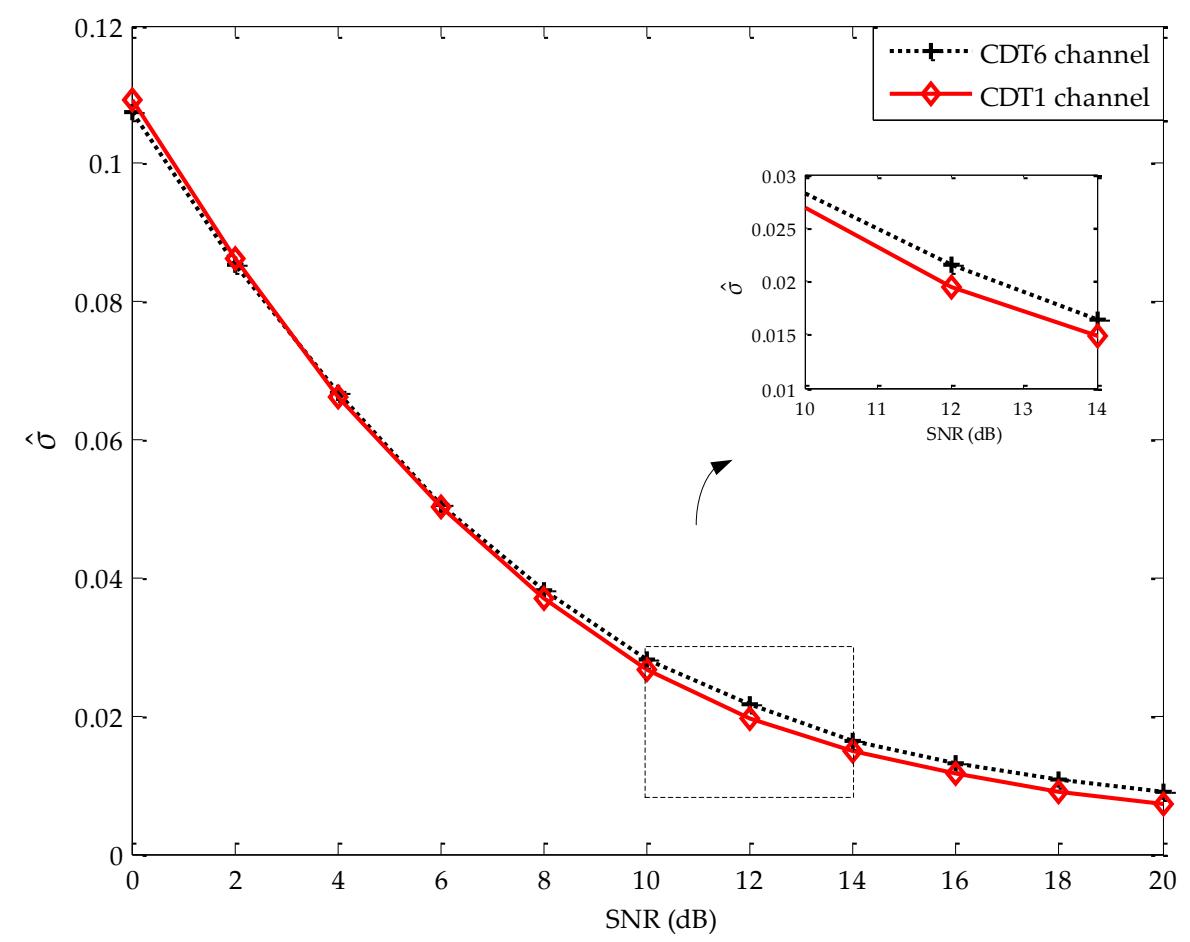

Figure 5. The threshold $\hat{\sigma}$ under CDT1 and CDT6 channels.

\subsection{The Performance in Static Channel}

The performance metrics BER and NMSE under static CDT1 channel are shown in Figures 6 and 7, respectively. The performance metrics BER and NMSE under static CDT6 channel are shown in Figures 8 and 9 , respectively.

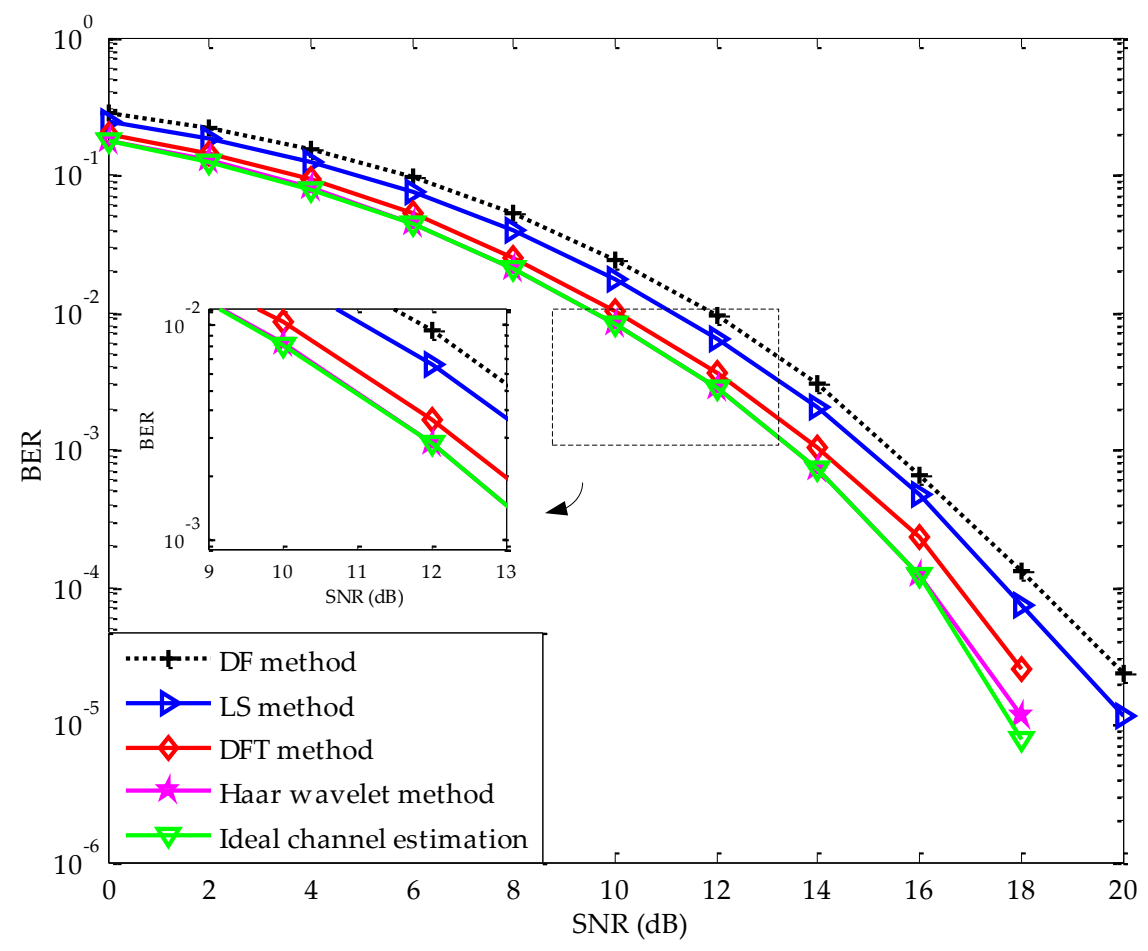

Figure 6. Bit error rate (BER) performance of quadrature phase shift keying (QPSK) modulated CP-OFDM and wavelet-OFDM systems under CDT1 channel. 
As can be seen from Figure 6, the BER performance of above several channel estimation methods all decreases with the increase of SNR. Under the same conditions, the DF and LS channel estimation methods have higher BER results among the four channel estimation methods. Although LS channel estimation method has about $0.3 \mathrm{~dB}$ gains compared with DF method at BER of $10^{-2}$, the DF method has high data transmission rate and spectrum efficiency. The proposed Haar wavelet method has the lowest BER among the four channel estimation methods. At the BER of $10^{-3}$, the proposed Haar wavelet method can provide about $0.5 \mathrm{~dB}$ and $1.5 \mathrm{~dB}$ SNR gains compared with DFT and DF methods, respectively. Hence, under static slight frequency-selectivity CDT1 channel conditions, the Haar wavelet method can be used to estimate the channel properties.

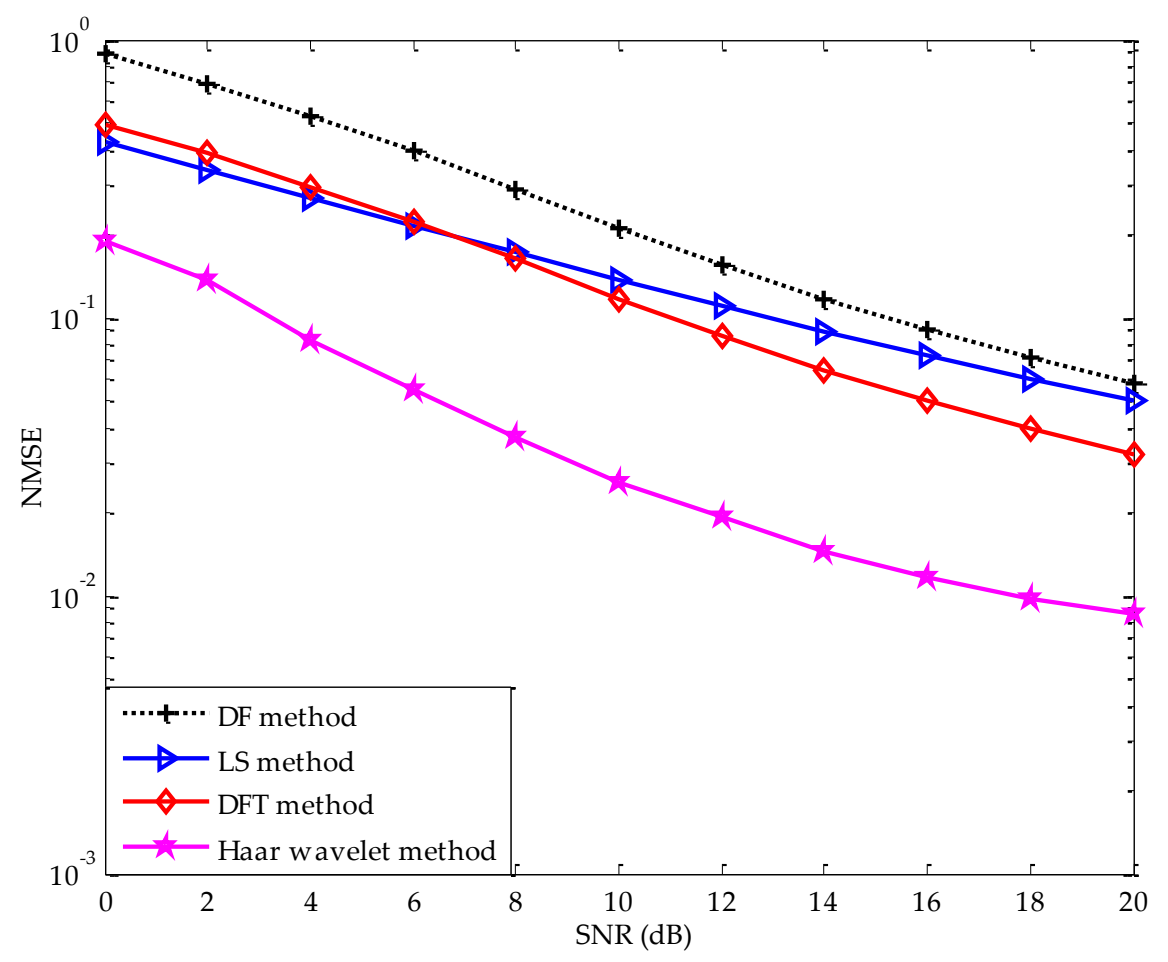

Figure 7. Normalized mean square error (NMSE) performance of QPSK modulated CP-OFDM and wavelet-OFDM systems under CDT1 channel.

As can be seen from Figure 7, as the SNR increases in the CP-OFDM and wavelet-OFDM systems, the simulation results of four channel estimation methods are decreased in terms of NMSE. Under the same conditions, the proposed Haar wavelet method has the lowest NMSE performance. At the NMSE of $10^{-1}$, compared with the DFT channel estimation method, the proposed Haar wavelet method has about $7.5 \mathrm{~dB}$ SNR gains.

As can be seen from Figures 8 and 9, the BER and NMSE performance are presented in CDT6 channel. The CDT6 channel has stronger frequency-selectivity than CDT1 channel. Hence, the performance under CDT6 channel is worse than CDT1 channel. With the increase of SNR, the simulation results show a trend of overall decreasing. Although the CDT6 channel has worse propagate condition than CDT1 channel, the proposed Haar wavelet method also has the best performance among the four channel estimation methods. In Figure 8, the simulation results about the proposed Haar wavelet method is very close to the ideal channel estimation. At the BER of $10^{-2}$, compared with the DFT, LS, and DF channel estimation methods, the proposed Haar wavelet method has about $0.9 \mathrm{~dB}, 2.5 \mathrm{~dB}$, and $3 \mathrm{~dB}$ BER gains, respectively. In Figure 9, compared with the DFT, LS, and DF methods, the proposed Haar wavelet method has about $8.4 \mathrm{~dB}, 10.3 \mathrm{~dB}$, and 13.1 dB NMSE gains at the NMSE of $10^{-1}$. Hence, the proposed Haar wavelet method has the best performance among the DF, LS, and DFT channel estimation methods. 


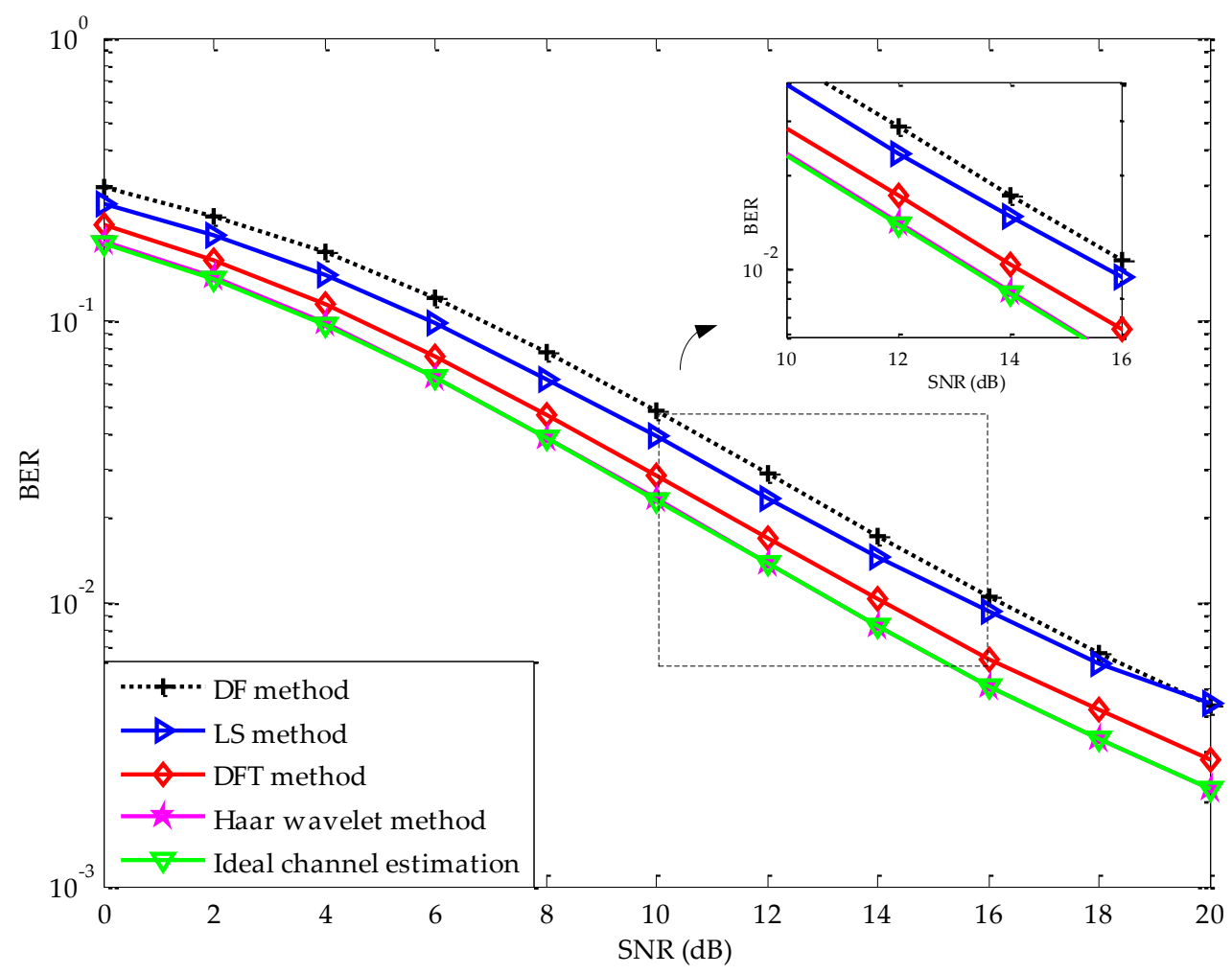

Figure 8. BER performance of QPSK modulated CP-OFDM and wavelet-OFDM systems under CDT6 channel.

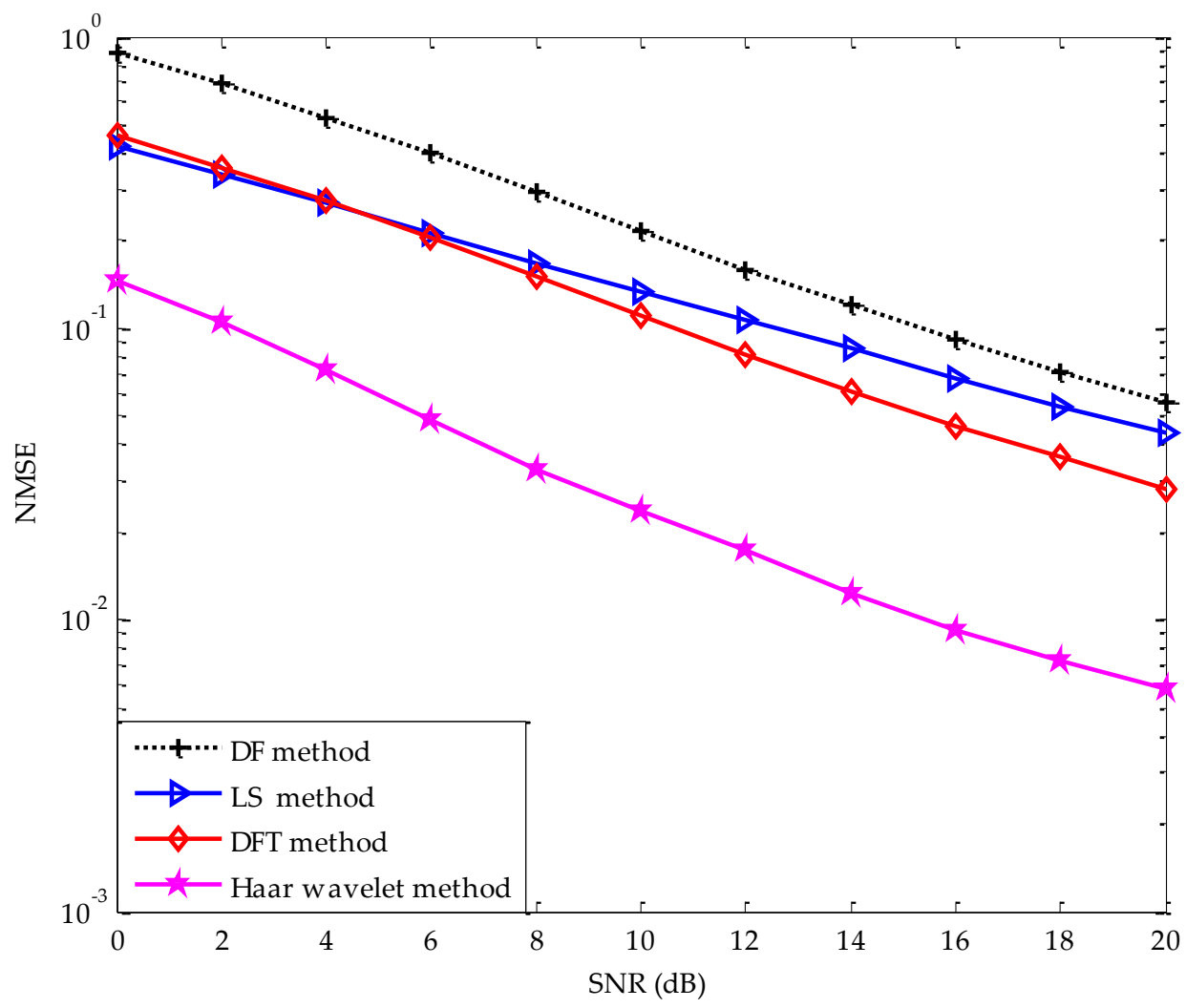

Figure 9. NMSE performance of QPSK modulated CP-OFDM and wavelet-OFDM systems under CDT6 channel. 


\subsection{The Performance in Dynamic Channel with Doppler Spread $20 \mathrm{~Hz}$}

The BER performances in CDT1 and CDT6 channels with Doppler spread $20 \mathrm{~Hz}$ are presented in Figures 10 and 11, respectively. The LS channel estimation method is simple to implement, but it is susceptible to the AWGN of the channel. The DFT channel estimation method just reduces the channel estimation noise in time-domain, but it cannot adapt to the time-varying channel. The DF and proposed Haar wavelet methods both use the previous one OFDM symbol's CFR as the current OFDM symbol's CFR. Hence, these two methods have large estimation errors when the channel is time variant. Therefore, under the condition of the Doppler spread equivalent to $20 \mathrm{~Hz}$, the BER and NMSE performance are worse than that in static channel.

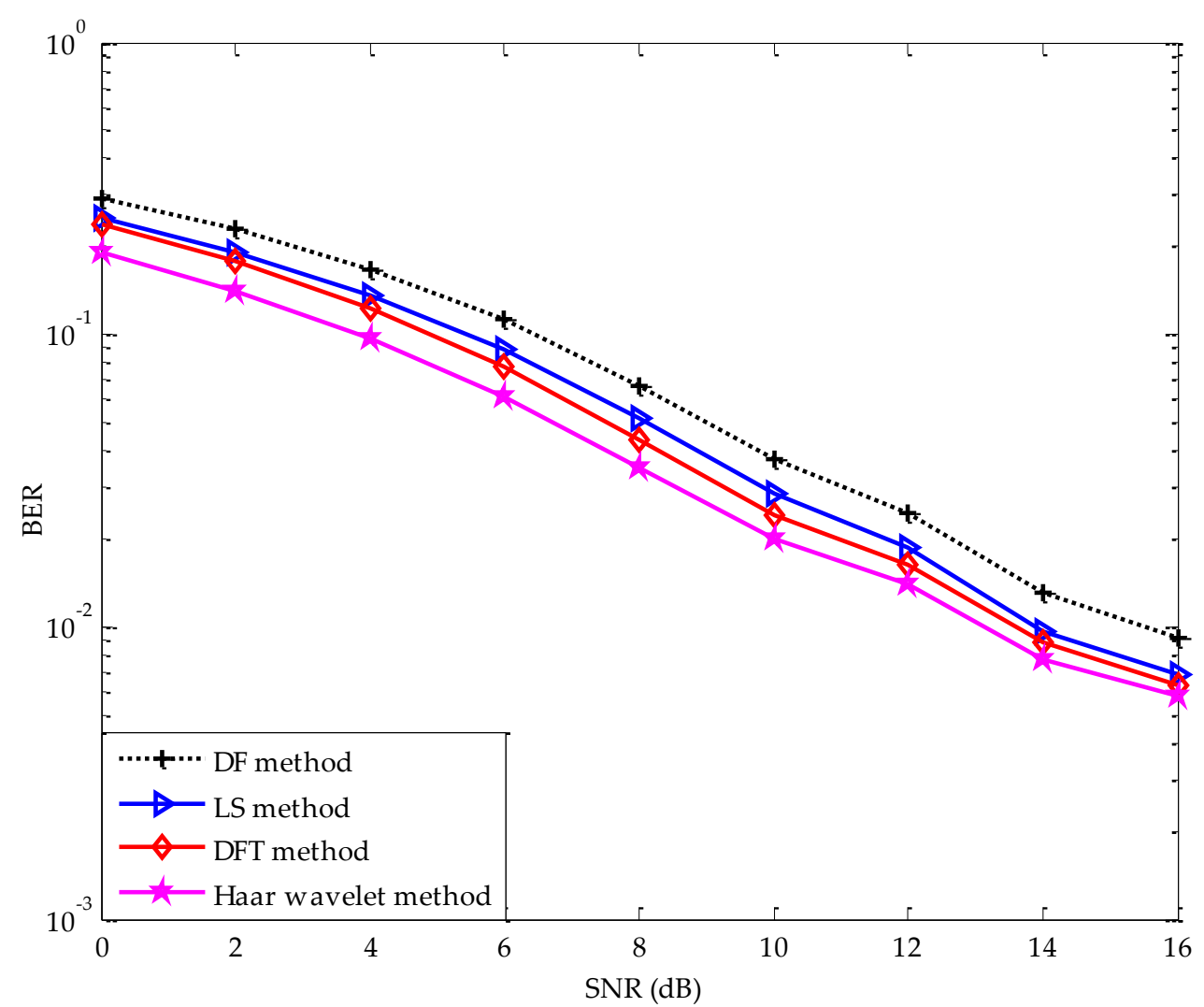

Figure 10. BER performance of QPSK modulated CP-OFDM and wavelet-OFDM systems under CDT1 channel with Doppler spread $20 \mathrm{~Hz}$.

As can be seen from Figure 10, different channel estimation methods have the same trend in terms of BER. With the increase of SNR, the simulation results show a trend of overall decreasing. The proposed Haar wavelet method has the lowest BER. The simulation results show that the proposed Haar wavelet method is significantly better than other conventional methods in the CDT1 channel with Doppler spread $20 \mathrm{~Hz}$. At the BER of $10^{-2}$, compared with the DFT, LS, and DF channel estimation methods, the proposed Haar wavelet method has about $0.45 \mathrm{~dB}, 0.7 \mathrm{~dB}$, and $2.35 \mathrm{~dB} B E R$ gains, respectively.

As can be seen from Figure 11, the proposed Haar wavelet method has the best performance among the four channel estimation methods under CDT6 channel with Doppler spread $20 \mathrm{~Hz}$. When the BER is $2 \times 10^{-2}$, compared with the DFT, LS, and DF channel estimation methods, the proposed Haar wavelet method has about $0.95 \mathrm{~dB}, 2.2 \mathrm{~dB}$, and $3.5 \mathrm{~dB}$ gains, respectively. Hence, the proposed Haar wavelet method has the best performance among the four channel estimation methods. 


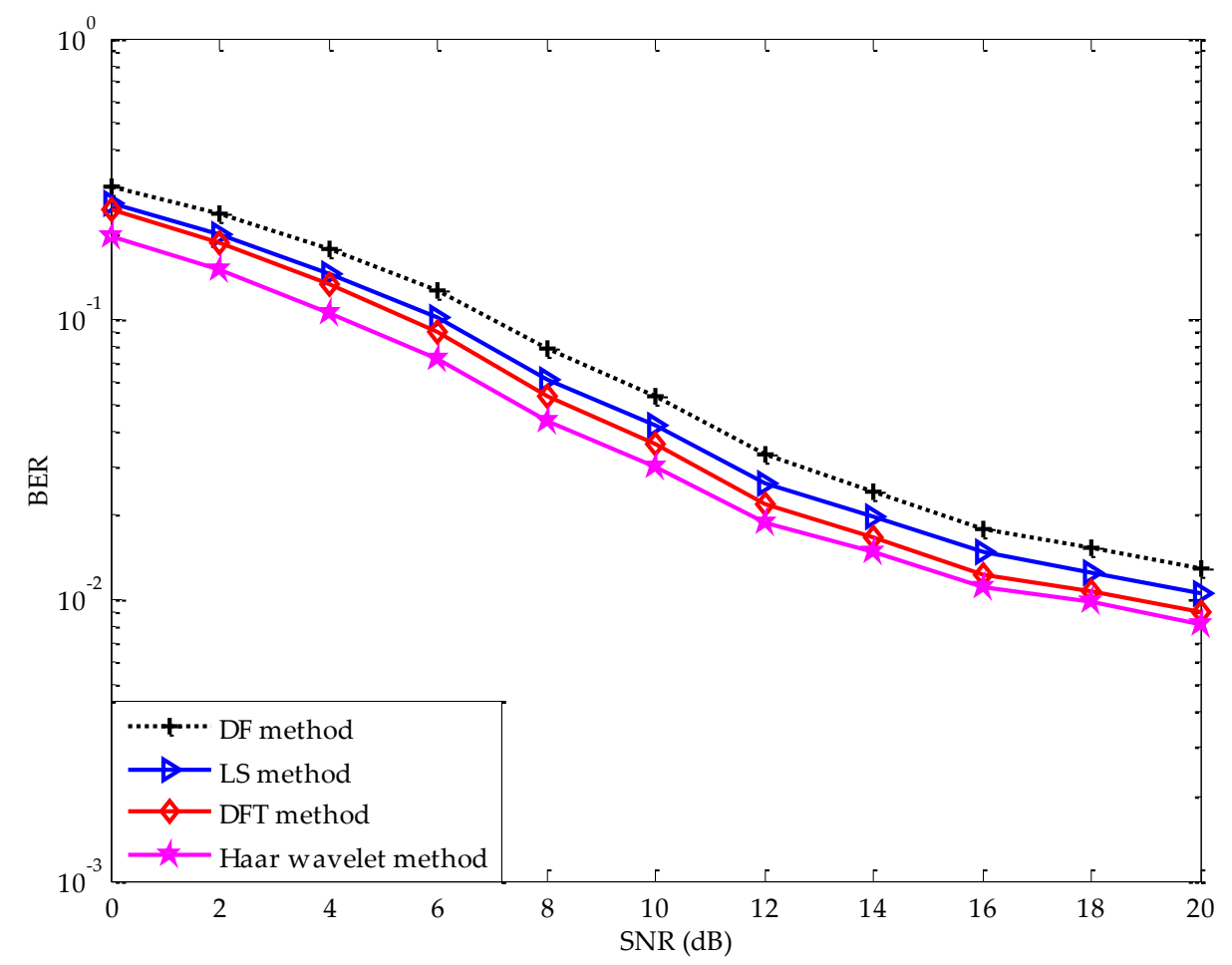

Figure 11. BER performance with QPSK modulated CP-OFDM and wavelet-OFDM systems under CDT6 channel with Doppler spread 20Hz.

\section{Conclusions and Future Work}

This paper proposes a Haar wavelet decision feedback channel estimation method. The DF channel estimation method can provide high data transmission rate and spectrum efficiency while the wavelet decomposition can suppress the AWGN existing at the estimated channel coefficients in time-domain effectively. Simulation results have verified that combining these two methods can provide good performance under static slight frequency-selectivity CDT1 channel. But under the conditions of strong frequency-selectivity and dynamic channels, the proposed Haar wavelet method needs improvement to further improve the accuracy of channel estimation.

Compared with the conventional DF channel estimation method, the proposed Haar wavelet method has better performance. Compared with the LS and DFT channel estimation methods, the proposed Haar wavelet method not only has better performance but also has high data transmission rate and spectrum efficiency. With the improvement of the fifth generation (5G) technology, there are more demands for high transmission data rate and spectrum efficiency in communication systems. Hence, the proposed Haar wavelet channel estimation method has broad market prospect and can be applied in optical communication, radar communication, and underwater acoustic communication [5].

In future work, we will do some significant researches on the following aspects. First, we will apply the Haar wavelet method in Turbo coded CP-OFDM system $[7,8]$ to better adapt to the strong frequency-selective and dynamic channels. Second, we will make a meaningful study about the application of other wavelets in channel estimation.

Author Contributions: R.T. and X.Z. conceived the algorithm and designed the experiments; R.T. performed the experiments; X.Z. and C.W. analyzed the results; R.T. drafted the manuscript; R.T., X.Z., and C.W. revised the manuscript. All authors read and approved the final manuscript.

Acknowledgments: This work was supported by the National Natural Science Foundation of China (No. 61702303) and the Natural Science Foundation of Shandong Province, China (No. ZR2017MF020, No. ZR2015PF004).

Conflicts of Interest: The authors declare no conflict of interest. 


\section{References}

1. Liu, Y.S.; Tan, Z.H.; Hu, H.J.; Cimini, L.J.; Li, G.Y. Channel estimation for OFDM. IEEE Commun. Surv. Tutor. 2014, 16, 1891-1908. [CrossRef]

2. Uwaechia, A.N.; Mahyuddin, N.M. Collaborative framework of algorithms for sparse channel estimation in OFDM systems. J. Commun. Netw. 2018, 20, 9-19.

3. Miao, P.; Chen, P.; Chen, Z.M. Low-complexity PAPR reduction scheme combining multi-band Hadamard precoding and clipping in OFDM-based optical communications. Electronics 2018, 7, 11. [CrossRef]

4. Elahi, A.; Qureshi, I.M.; Khan, Z.U.; Zaman, F. Sidelobe reduction in non-contiguous OFDM-based cognitive radio systems using a generalized sidelobe canceller. Appl. Sci. 2015, 5, 894-909. [CrossRef]

5. Ashri, R.; Shaban, H.; El-Nasr, M.A. A novel fractional Fourier transform-based ASK-OFDM system for underwater acoustic communications. Appl. Sci. 2017, 7, 1286. [CrossRef]

6. Bölcskei, H.; Gesbert, D.; Paulraj, A.J. On the capacity of OFDM-based spatial multiplexing systems. IEEE Trans. Commun. 2002, 50, 225-234. [CrossRef]

7. Chronopoulos, S.K.; Tatsis, G.; Kostarakis, P. Turbo coded OFDM with large number of subcarriers. JSIP 2012, 3, 161-168. [CrossRef]

8. Chronopoulos, S.K.; Christofilakis, V.; Tatsis, G.; Kostarakis, P. Performance of Turbo coded OFDM under the presence of various noise types. Wirel. Pers. Commun. 2016, 87, 1319-1336. [CrossRef]

9. Lin, T.T.; Hwang, F.H. On the design of an elegant CFO estimate system with the assistance of pilots for OFDM transmission. Appl. Sci. 2015, 5, 1773-1789. [CrossRef]

10. Beek, J.J.; Edfors, O.; Sandell, M.; Wilson, S.K.; Borjesson, P.O. On channel estimation in OFDM systems. In Proceedings of the IEEE 45th Vehicular Technology Conference, Chicago, IL, USA, 25-28 July 1995; pp. 815-819.

11. Faradina, N.; Iskandar. Pilot based LS channel estimation for downlink HAPS channel. In Proceedings of the 1st International Symposium on Electronics and Smart Devices, Bandung, Indonesia, 29-30 November 2016; pp. 355-359.

12. Li, G.M.; Liao, G.S. A pilot-pattern based algorithm for MIMO-OFDM channel estimation. Algorithms 2017, 10, 3. [CrossRef]

13. Liu, K.W.; Xing, K. Research of MMSE and LS channel estimation in OFDM systems. In Proceedings of the 2nd International Conference on Information Science and Engineering, Hangzhou, China, 4-6 December 2010; pp. 2308-2311.

14. Noh, M.; Lee, Y.; Park, H. Low complexity LMMSE channel estimation for OFDM. IEE Proc. Commun. 2006, 153, 645-650. [CrossRef]

15. Desai, M.V.; Gupta, S.; Dalal, U.D. DCT-SVD based channel estimation technique in IEEE 802.16e DL-PUSC system. In Proceedings of the 2nd International Conference on Emerging Technology Trends in Electronics, Communication and Networking, Surat, India, 26-27 December 2014; pp. 1-6.

16. Lee, Y.S.; Shin, H.C.; Kim, H.N. Channel estimation based on a time-domain threshold for OFDM systems. IEEE Trans. Broadcast. 2009, 55, 656-662.

17. Zhou, X.; Ye, Z.; Liu, X.X.; Wang, C.Y. Channel estimation based on linear filtering least square in OFDM systems. J. Commun. 2016, 11, 1005-1011. [CrossRef]

18. Tseng, C.H.; Cheng, Y.C.; Chung, C.D. Subspace-based blind channel estimation for OFDM by exploiting cyclic prefix. IEEE Wirel. Commun. Lett. 2013, 2, 691-694. [CrossRef]

19. Wang, H.; Liao, J.Q.; Xu, L.W.; Wang, X.P. Blind channel estimation for FBMC/OQAM systems based on subspace approach. Information 2018, 9, 58. [CrossRef]

20. Mezghani, A.; Swindlehurst, A.L. Blind estimation of sparse broadband massive MIMO channels with ideal and one-bit ADCs. IEEE Trans. Signal Process. 2018, 66, 2972-2983. [CrossRef]

21. Zhang, W.L.; Gao, F.F.; Yin, Q.Y. Blind channel estimation for MIMO-OFDM systems with low order signal constellation. IEEE Commun. Lett. 2015, 19, 499-502. [CrossRef]

22. Shirmohammadi, M.; Mohammad, A.D. Blind channel estimation of MIMO-OFDM systems in satellite communication. In Proceedings of the 6th International Conference on Information and Communication Technology Convergence, Jeju Island, Korea, 28-30 October 2015; pp. 704-709. 
23. Ghadrdan, S.; Ahmadian, M.; Salari, S.; Heydarzadeh, M. An improved blind channel estimation algorithm for OFDM systems. In Proceedings of the 5th International Symposium on Telecommunications, Tehran, Iran, 4-6 December 2010; pp. 421-425.

24. Ladaycia, A.; Mokraoui, A.; Abed-Meraim, K.; Belouchrani, A. Performance bounds analysis for semi-blind channel estimation in MIMO-OFDM communications systems. IEEE Trans. Wirel. Commun. 2017, 16, 5925-5938. [CrossRef]

25. Liu, K.F.; Da Costa, J.P.C.L.; So, H.C.; De Almeida, A.L.F. Semi-blind receivers for joint symbol and channel estimation in space-time-frequency MIMO-OFDM systems. IEEE Trans. Signal Process. 2013, 61, 5444-5457. [CrossRef]

26. Nayebi, E.; Rao, B.D. Semi-blind channel estimation for multiuser massive MIMO-OFDM systems. IEEE Trans. Signal Process. 2018, 66, 540-553. [CrossRef]

27. Ban, Y.R.; Hu, Q.; Mao, Z.D.; Zhao, Z.Y. Semi-blind pilot-aided channel estimation in uplink cloud radio access networks. China Commun. 2016, 13, 72-79. [CrossRef]

28. Mawatwal, K.; Sen, D.; Roy, R. A semi-blind channel estimation algorithm for massive MIMO systems. IEEE Wirel. Commun. Lett. 2017, 6, 70-73. [CrossRef]

29. Gulomjon, S.; Fu, Y.Q.; Sangirov, J.; Ye, F.; Olmasov, A. A performance analysis of optimized semi-blind channel estimation method in OFDM systems. In Proceedings of the 19th International Conference on Advanced Communications Technology, Pyeongchang, Korea, 19-22 February 2017; pp. 907-912.

30. Medles, A.; Slock, D.T.M.; De Carvalho, E. Linear prediction based semi-blind estimation of MIMO FIR channels. In Proceedings of the 3rd IEEE Workshop on Signal Processing Advances in Wireless Communications, Taiwan, China, 20-23 March 2001; pp. 58-61.

31. Ma, J.; Yu, H.; Liu, S.Y. The MMSE channel estimation based on DFT for OFDM system. In Proceedings of the 5th International Conference on Wireless Communications, Networking and Mobile Computing, Beijing, China, 24-26 September 2009; pp. 1-4.

32. Ran, J.J.; Grünheid, R.; Rohling, H.; Bolinth, E.; Kern, R. Decision-directed channel estimation method for OFDM systems with high velocities. In Proceedings of the 57th IEEE Semiannual Vehicular Technology Conference, Jeju, Korea, 22-25 April 2003; pp. 2358-2361.

33. Oltean, M.; Naforniţă, M. Errors per scale statistics for a wavelet OFDM transmission in flat fading channels. In Proceedings of the 6th IEEE International Symposium on Intelligent Signal Processing, Budapest, Hungary, 26-28 August 2009; pp. 119-124.

34. Kattoush, A.H.; Mahmoud, W.A.; Nihad, S. The performance of multiwavelets based OFDM system under different channel conditions. Digit. Signal Process. Rev. J. 2010, 20, 472-482. [CrossRef]

35. Chandra, K.S.; Hussain, S.; Fernando, X. Wavelet based OFDM for power line communication. In Proceedings of the IEEE 27th Canadian Conference on Electrical and Computer Engineering, Toronto, Canada, 4-7 May 2014; pp. 1-4.

36. Liu, W.F.; Pan, B.W. Research on channel estimation for OFDM system with wavelet de-noising (in Chinese). Comput. Simul. 2013, 30, 185-188.

37. Zhou, X.; Yang, F.; Song, J. Novel transmit diversity scheme for TDS-OFDM system with frequency-shift m-sequence padding. IEEE Trans. Broadcast. 2012, 58, 317-324. [CrossRef]

(C) 2018 by the authors. Licensee MDPI, Basel, Switzerland. This article is an open access article distributed under the terms and conditions of the Creative Commons Attribution (CC BY) license (http://creativecommons.org/licenses/by/4.0/). 